\title{
Indications on the Higgs boson mass from lattice simulations
}

\author{
P. Cea ${ }^{\mathrm{a}}$, M. Consoli ${ }^{\mathrm{b}}$ and L. Cosmai ${ }^{\mathrm{c}}$

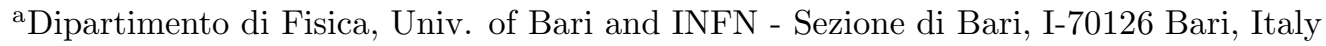 \\ bINFN - Sezione di Catania, I-70126 Catania, Italy \\ ${ }^{\mathrm{c} I N F N ~-~ S e z i o n e ~ d i ~ B a r i, ~ I-70126 ~ B a r i, ~ I t a l y ~}$
}

The 'triviality' of $\Phi_{4}^{4}$ has been traditionally interpreted within perturbation theory where the prediction for the Higgs boson mass depends on the magnitude of the ultraviolet cutoff $\Lambda$. This approach crucially assumes that the vacuum field and its quantum fluctuations rescale in the same way. The results of the present lattice simulation, confirming previous numerical indications, show that this assumption is not true. As a consequence, large values of the Higgs mass $m_{H}$ can coexist with the limit $\Lambda \rightarrow \infty$. As an example, by extrapolating to the Standard Model our results obtained in the Ising limit of the one-component theory, one can obtain a value as large as $m_{H}=760 \pm 21 \mathrm{GeV}$, independently of $\Lambda$.

Traditionally, the 'triviality' of $\Phi^{4}$ theories in $3+1$ space-time dimensions [1] has been interpreted within perturbation theory. In this interpretation, these theories represent just an effective description, valid only up to some cutoff scale $\Lambda$. This conventional view, when used in the Standard Model, leads to predict that the Higgs boson mass squared, $m_{H}^{2}$, is proportional to $g_{R} v_{R}^{2}$, where $v_{R}$ is the known weak scale $(246 \mathrm{GeV})$ and $g_{R} \sim 1 / \ln \Lambda$ is the renormalized scalar self-coupling. Therefore, the ratio $m_{H} / v_{R}$ would be a cutoff-dependent quantity that becomes smaller and smaller when $\Lambda$ is made larger and larger. However, in an alternative approach $[23$ this conclusion is not true. The 'Higgs condensate' and its quantum fluctuations undergo different rescalings when changing the ultraviolet cutoff, so that the relation between $m_{H}$ and the physical $v_{R}$ is not the same as in perturbation theory.

To understand this point, we observe that beyond perturbation theory, in a broken-symmetry phase, there are two different definitions of the field rescaling. There is a rescaling of the 'condensate', say $Z \equiv Z_{\varphi}$, and a rescaling of the fluctuations, say $Z \equiv Z_{\text {prop }}$. Consider a one-component scalar theory and introduce the bare expectation value $v_{B}=\left\langle\Phi_{\text {latt }}\right\rangle$ associated with the 'lattice' field as defined at the cutoff scale. By $Z \equiv Z_{\varphi}$ we mean the rescaling that is needed to obtain the physical vacuum field $v_{R}=v_{B} / \sqrt{Z_{\varphi}}$. Since the second derivative of the effective potential is the zero-four-momentum two-point function, this standard definition is equivalent to define $Z_{\varphi}$ as:

$Z_{\varphi}=m_{H}^{2} \chi_{2}(0)$

where $\chi_{2}(0)$ is the zero-momentum susceptibility. On the other hand, $Z \equiv Z_{\text {prop }}$ is determined from the residue of the connected propagator on its mass shell. Assuming 'triviality' and the KállenLehmann representation for the shifted quantum field, one predicts $Z_{\text {prop }} \rightarrow 1$ when approaching the continuum theory.

Now, in the standard approach one assumes $Z_{\varphi} \sim Z_{\text {prop }}$ while in the different interpretation of triviality, 23] although $Z_{\text {prop }} \rightarrow 1$, as in leadingorder perturbation theory, $Z_{\varphi} \sim \ln \Lambda$ is fully non perturbative and diverges in the continuum limit. In this case, in order to obtain $v_{R}$ from the bare $v_{B}$ one has to apply a non-trivial correction. As a result, $m_{H}$ and $v_{R}$ now scale uniformly in the continuum limit, and the ratio $C=m_{H} / v_{R}$ is a cutoff-independent quantity. To check this alternative picture against the generally accepted point of view, one can run numerical simulations 
of the theory. In this respect, we observe that numerical evidence for different cutoff dependencies of $Z_{\varphi}$ and $Z_{\text {prop }}$ has already been already reported 456. In those calculations, one was fitting the lattice data for the connected propagator to the (lattice version of the) two-parameter form

$G_{\text {fit }}(p)=\frac{Z_{\text {prop }}}{p^{2}+m_{\text {latt }}^{2}}$

After computing the zero-momentum susceptibility $\chi_{\text {latt }}$, it was possible to compare the value of $Z_{\varphi} \equiv m_{\text {latt }}^{2} \chi_{\text {latt }}$ with the fitted $Z_{\text {prop }}$, both in the symmetric and broken phases. While no difference was found in the symmetric phase, $Z_{\varphi}$ and $Z_{\text {prop }}$ were found to be sizeably different in the broken phase. $Z_{\text {prop }}$ was very slowly varying and steadily approaching unity from below in the continuum limit. On the other hand, $Z_{\varphi}$ was found to rapidly increase above unity in the same limit. A possible objection to this strategy is that the two-parameter form Eq. (2), although providing a good description of the lattice data, neglects higher-order corrections to the structure of the propagator. As a consequence, one might object that the extraction of the various parameters is affected in an uncontrolled way. For this reason, we have decided to change strategy by performing a new set of lattice calculations. Rather than studying the propagator, we have addressed the model-independent lattice measurement of the susceptibility. In this way, assuming the mass values from perturbation theory, one can obtain a precise determination of $Z_{\varphi}$ to be compared with the perturbative predictions.

The numerical simulations were performed in the Ising limit where a one-component $\left(\lambda \Phi^{4}\right)_{4}$ theory becomes

$S_{\text {Ising }}=-\kappa \sum_{x} \sum_{\mu}\left[\phi\left(x+\hat{e}_{\mu}\right) \phi(x)+\phi\left(x-\hat{e}_{\mu}\right) \phi(x)\right]$

and $\phi(x)$ takes only the values \pm 1 (in an infinite lattice, the broken phase is found for $\kappa>$ 0.07475). Using the Swendsen-Wang and Wolff cluster algorithms we have computed the zero- momentum susceptibility:

$\chi_{\text {latt }}=L^{4}\left[\left\langle|\phi|^{2}\right\rangle-\langle|\phi|\rangle^{2}\right]$.

We used different lattice sizes at each value of $\kappa$ to have a check of the finite-size effects. Statistical errors have been estimated using the jackknife. Finally, we have checked our results with those obtained by other authors 7 .

As anticipated, we shall use the perturbative predictions for the Higgs boson mass adopting the Lüscher-Weisz scheme 8 . To this end, let us denote by $m_{\text {input }}$ the value of the parameter $m_{R}$ reported in the first column of Table 3 in Ref. 8 for any value of $\kappa$ (the Ising limit corresponding to the value of the other parameter $\bar{\lambda}=1$ ). In this way, one can compare the quantity

$Z_{\varphi} \equiv 2 \kappa m_{\text {input }}^{2} \chi_{\text {latt }}$

with the perturbative prediction for $Z_{\mathrm{LW}} \equiv 2 \kappa Z_{R}$ where $Z_{R}$ is defined in the third column of Table 3 in Ref. 8.

The values of $Z_{\varphi}$ and $Z_{\mathrm{LW}}$ for various $\kappa$ are reported in Fig.1. As one can check, the two $Z$ 's follow completely different trends and the discrepancy becomes larger and larger when approaching the continuum limit, precisely the same trend found in Refs. 56. This confirms that, approaching the continuum limit, the rescaling of the 'Higgs condensate' cannot be described in perturbation theory. In addition, the lattice data for $Z_{\varphi}$ are completely consistent with the alternative scenario $\sim \ln \Lambda$ predicted in Refs. 23].

Now, if the physical $v_{R}$ has to be computed from the bare $v_{B}$ through $Z=Z_{\varphi}$, rather than through the perturbative $Z=Z_{\mathrm{LW}}$, one may wonder about the $m_{H}-v_{R}$ correlation. In this case the perturbative relation

$\left[\frac{m_{H}}{v_{R}}\right]_{\mathrm{LW}} \equiv \sqrt{\frac{g_{R}}{3}}$.

becomes

$\frac{m_{H}}{v_{R}}=\sqrt{\frac{g_{R}}{3} \frac{Z_{\varphi}}{Z_{\mathrm{LW}}}} \equiv C$

obtained by replacing $Z_{\mathrm{LW}} \rightarrow Z_{\varphi}$ in Ref. 8] but correcting for the perturbative $Z_{\mathrm{LW}}$ introduced 


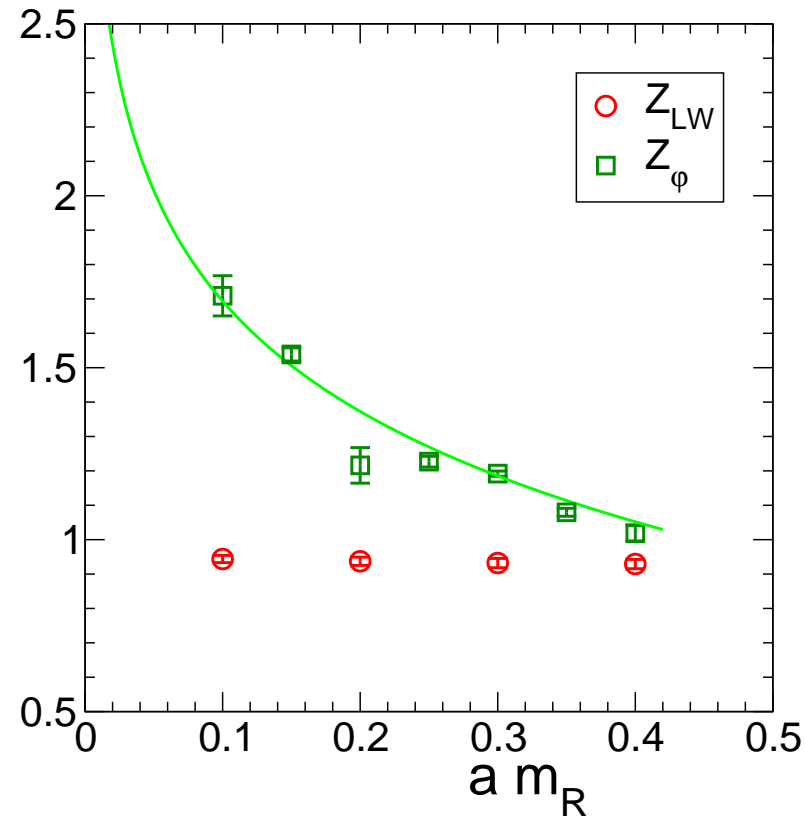

Figure 1. The values of $Z_{\varphi}$ as defined through Eq. (5) and $Z_{\mathrm{LW}}$ versus $m_{\text {input }}=a m_{R}$. Solid line is the fit of $Z_{\varphi}$ according to $Z_{\varphi}=$ constant + $B \ln \frac{1}{a m_{R}}$.

in the Lüscher and Weisz approach. Assuming the values of $g_{R}$ reported in the second column of Table 3 of Ref. [8] and using our values of $Z_{\varphi}$, we have reported in Fig.2 the values of $m_{H}$ as defined through Eq. (7) versus $m_{\text {input }}=a m_{R}$ for $v_{R}=246 \mathrm{GeV}$. The error band corresponds to a one standard deviation error in the determination of $m_{H}$ through a fit with a constant function. As one can see, the $Z_{\varphi} \sim \ln \Lambda$ trend observed in Fig.1, compensates the $1 / \ln \Lambda$ from $g_{R}$ so that $C$ turns out to be a cutoff-independent constant.

Our results imply that the value of the Higgs boson mass, in units of $246 \mathrm{GeV}$, does not depend on the magnitude of the ultraviolet cutoff. Therefore, the whole issue of the upper bounds on the Higgs mass is affected suggesting the need of more extensive studies of the critical line to compare the possible values of $C$ in the full 2parameter $\Phi_{4}^{4}$ theory.

In any case, a value as large as $m_{H}=760 \pm$ $21 \mathrm{GeV}$, would also be in good agreement with

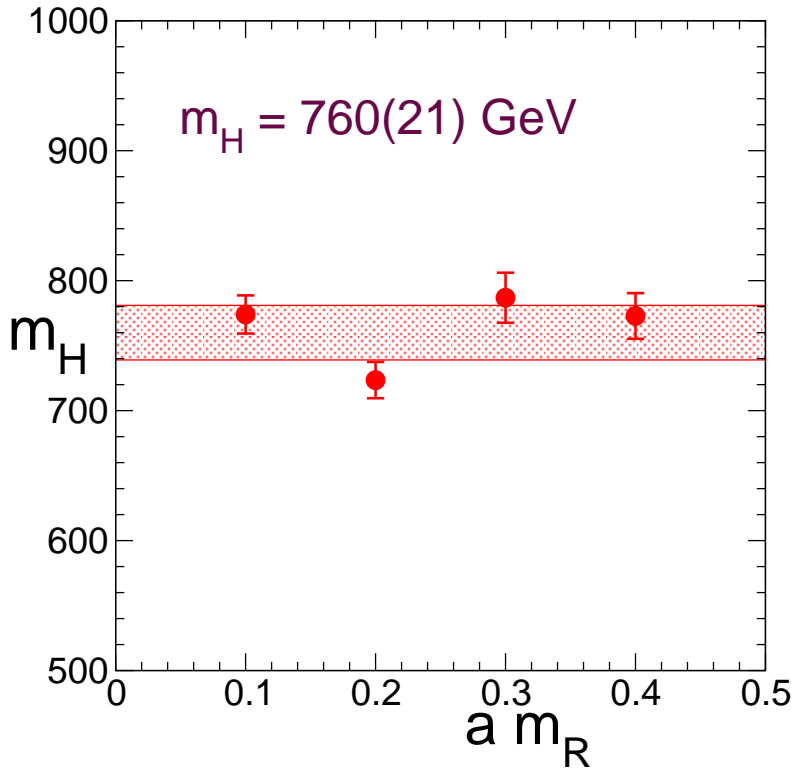

Figure 2. $m_{H}$ as defined through Eq. (17) versus $m_{\text {input }}=a m_{R}$

a recent phenomenological analysis of radiative corrections [9] that points toward substantially larger Higgs masses than previously obtained through global fits to Standard Model observables.

\section{REFERENCES}

1. R. Fernandez, J. Fröhlich and A.D. Sokal, Random Walks, Critical Phenomena, and Triviality in Quantum Field Theory (Springer-Verlag, Berlin, 1992).

2. M. Consoli and P.M. Stevenson, Z. Phys. C63 (1994) 427, hep-ph/9310338

3. M. Consoli and P.M. Stevenson, Int. J. Mod. Phys. A15 (2000) 133, hep-ph/9905427

4. P. Cea, M. Consoli and L. Cosmai, Mod. Phys. Lett. A13 (1998) 2361, hep-lat/9805005.

5. P. Cea et al., Mod. Phys. Lett. A14 (1999) 1673, hep-lat/9902020

6. P. Cea, M. Consoli and L. Cosmai, Nucl. Phys. Proc. Suppl. 83 (2000) 658, hep-lat/9909055.

7. K. Jansen et al., Nucl. Phys. B322 (1989) 
698.

8. M. Luscher and P. Weisz, Nucl. Phys. B295 (1988) 65.

9. W. Loinaz et al., Phys. Rev. D67 (2003) 073012, hep-ph/0210193. 